Washington University School of Medicine Digital Commons@Becker

$1-1-2021$

\title{
Diffusion basis spectrum imaging measures anti-inflammatory and neuroprotective effects of fingolimod on murine optic neuritis
}

\author{
Ruimeng Yang \\ Washington University School of Medicine in St. Louis \\ Tsen-Hsuan Lin \\ Washington University School of Medicine in St. Louis \\ Jie Zhan \\ Washington University School of Medicine in St. Louis \\ Shengsheng Lai \\ Guangdong Food and Drug Vocational College \\ Chunyu Song \\ Washington University School of Medicine in St. Louis
}

See next page for additional authors

Follow this and additional works at: https://digitalcommons.wustl.edu/open_access_pubs

Please let us know how this document benefits you.

\author{
Recommended Citation \\ Yang, Ruimeng; Lin, Tsen-Hsuan; Zhan, Jie; Lai, Shengsheng; Song, Chunyu; Sun, Peng; Ye, Zezhong; \\ Wallendorf, Michael; George, Ajit; Cross, Anne H; and Song, Sheng-Kwei, "Diffusion basis spectrum \\ imaging measures anti-inflammatory and neuroprotective effects of fingolimod on murine optic neuritis." \\ Neuroimage: Clinical. 31, 102732 (2021). \\ https://digitalcommons.wustl.edu/open_access_pubs/10647
}

This Open Access Publication is brought to you for free and open access by Digital Commons@Becker. It has been accepted for inclusion in Open Access Publications by an authorized administrator of Digital Commons@Becker. For more information, please contact vanam@wustl.edu. 


\section{Authors}

Ruimeng Yang, Tsen-Hsuan Lin, Jie Zhan, Shengsheng Lai, Chunyu Song, Peng Sun, Zezhong Ye, Michael Wallendorf, Ajit George, Anne H Cross, and Sheng-Kwei Song 


\title{
Diffusion basis spectrum imaging measures anti-inflammatory and neuroprotective effects of fingolimod on murine optic neuritis
}

\author{
Ruimeng Yang ${ }^{\mathrm{a}, \mathrm{b}, 1}$, Tsen-Hsuan Lin ${ }^{\mathrm{b}, 1}$, Jie Zhan ${ }^{\mathrm{b}}$, Shengsheng Lai ${ }^{\mathrm{c}}$, Chunyu Song ${ }^{\mathrm{b}}$, Peng Sun ${ }^{\mathrm{b}}$, \\ Zezhong Ye $^{\mathrm{b}}$, Michael Wallendorf ${ }^{\mathrm{d}}$, Ajit George ${ }^{\mathrm{b}}$, Anne H. Cross ${ }^{\mathrm{e}, \mathrm{f}}$, Sheng-Kwei Song ${ }^{\mathrm{b}, e, g, *}$

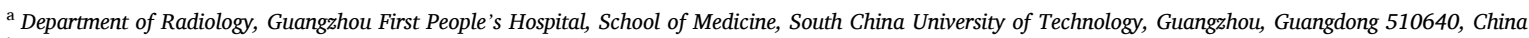 \\ ${ }^{\mathrm{b}}$ Department of Radiology, Washington University School of Medicine, St. Louis, MO 63110, USA \\ ${ }^{\mathrm{c}}$ Department of Medical Equipment, Guangdong Food and Drug Vocational College, Guangzhou, Guangdong 510520, China \\ d Department of Biostatistics, Washington University School of Medicine, St. Louis, MO 63110, USA \\ ${ }^{\mathrm{e}}$ Department of Neurology, Washington University School of Medicine, St. Louis, MO 63110, USA \\ ${ }^{\mathrm{f}}$ Hope Center for Neurological Disorders, Washington University School of Medicine, St. Louis, MO 63110, USA \\ ${ }^{\mathrm{g}}$ Department of Biomedical Engineering, Washington University in St. Louis, St. Louis, MO 63130, USA
}

\section{A R T I C L E I N F O}

\section{Keywords:}

Optic neuritis

Demyelination

Multiple sclerosis, diffusion basis spectrum

imaging

Axonal loss

Fingolimod

\begin{abstract}
A B S T R A C T
Objective: To prospectively determine whether diffusion basis spectrum imaging (DBSI) detects, differentiates and quantitates coexisting inflammation, demyelination, axonal injury and axon loss in mice with optic neuritis (ON) due to experimental autoimmune encephalomyelitis (EAE), and to determine if DBSI accurately measures effects of fingolimod on underlying pathology.

Methods: EAE was induced in 7-week-old C57BL/6 female mice. Visual acuity (VA) was assessed daily to detect onset of ON after which daily oral-treatment with either fingolimod $(1 \mathrm{mg} / \mathrm{kg})$ or saline was given for ten weeks. In vivo DBSI scans of optic nerves were performed at baseline, 2-, 6- and 10-weeks post treatment. DBSI-derived metrics including restricted isotropic diffusion tensor fraction (putatively reflecting cellularity), non-restricted isotropic diffusion tensor fraction (putatively reflecting vasogenic edema), DBSI-derived axonal volume, axial diffusivity, $\lambda_{\|}$(putatively reflecting axonal integrity), and increased radial diffusivity, $\lambda_{\perp}$ (putatively reflecting demyelination). Mice were killed immediately after the last DBSI scan for immunohistochemical assessment. Results: Optic nerves of fingolimod-treated mice exhibited significantly better $(p<0.05)$ VA than saline-treated group at each time point. During ten-week of treatment, DBSI-derived non-restricted and restricted-isotropicdiffusion-tensor fractions, and axonal volumes were not significantly different $(p>0.05)$ from the baseline values in fingolimod-treated mice. Transient DBSI- $\lambda_{\|}$decrease and DBSI- $\lambda_{\perp}$ increase were detected during Fingolimod treatment. DBSI-derived metrics assessed in vivo significantly correlated $(p<0.05)$ with the corresponding histological markers.

Conclusion: DBSI was used to assess changes of the underlying optic nerve pathologies in EAE mice with ON, exhibiting great potential as a noninvasive outcome measure for monitoring disease progression and therapeutic efficacy for MS.
\end{abstract}

\section{Introduction}

Multiple sclerosis (MS) is an immune-mediated inflammatory demyelinating disease of central nervous system (CNS). Irreversible axonal injury and axon loss are believed to be major causes of permanent neurologic impairments in MS (Rahim et al., 2018; Sun et al., 2020).
Although early intervention with disease-modifying therapy (DMT) has been shown to decrease the relapse rate and slow short-term disability progression in MS patients (Montalban et al., 2018), it is still uncertain whether it is associated with a lower long-term risk of disability (Kavaliunas et al., 2017; Torkildsen et al., 2016). To address this issue, robust markers which can precisely capture and discriminate axonal loss

\footnotetext{
* Corresponding author at: Biomedical MR Laboratory, Campus Box 8227, Washington University School of Medicine, Room 2313, 4525 Scott Ave, St Louis, MO 63110, USA.

E-mail address: ssong@wustl.edu (S.-K. Song).

${ }^{1}$ Ruimeng Yang and Tsen-Hsuan Lin contributed equally to this work.
} 
from other underlying pathologies, such as inflammation and demyelination, and monitor intervention response are urgently needed to help clinicians make more efficient personalized management decision for MS patients and to assess responses to treatments.

Optic neuritis (ON) is a frequent manifestation of MS, and is also frequently encountered on its own, or as part of other diseases such as neuromyelitis optica and myelin oligodendrocyte glycoprotein antibody disease. Conventional $\mathrm{T}_{2}$-weighted imaging, $\mathrm{T}_{1}$-weighted gadolinium contrast enhanced imaging, magnetization transfer imaging (MTI), and diffusion tensor imaging (DTI) have been widely applied on the evaluation of optic nerve in MS-related ON (Hickman et al., 2004, 2005; Jackson et al., 1998; Naismith et al., 2012a; Rocca and Filippi, 2007). In addition, optic nerve damages in patients with MS have been commonly assessed based on optical coherence tomography (OCT) measured retinal nerve fiber layer thickness change (Gajamange et al., 2018).

A lack of pathological specificity of these imaging modalities has contributed to discrepancies between neuroimaging findings and clinical outcomes, commonly known as the clinicoradiological paradox (Barkhof, 2002; Costello et al., 2006; Davis, 2014), resulting in part from the heterogeneous nature of MS in clinical course and underlying pathologies (Leray et al., 2010; Scalfari et al., 2010). We have developed diffusion basis spectrum imaging (DBSI) and demonstrated its ability to detect and differentiate inflammation, axonal injury, axon loss and demyelination in mice with experimental autoimmune encephalomyelitis (EAE), and autopsied and biopsied specimens from MS patients (Lin et al., 2017; Shirani et al., 2019; Sun et al., 2020; Wang et al., 2014, 2015, 2011; Ye et al., 2020).

Therefore, we hypothesized that DBSI, which is pathologically more specific than standard MRI, would be able to assess the efficacy of fingolimod (also known as FTY720, Selleck Chemicals LLC,) treatment of ON through longitudinally quantifying the evolution of several coexisting optic nerve pathologies using a preclinical EAE mouse model which has ON.

\section{Materials and Methods}

\subsection{Animal model}

Procedures were approved by Washington University Institutional Animal Care and Use Committee. Female C57BL/6 mice at 7 weeks of age were obtained from Jackson Laboratory (Bar Harbor, ME).

\subsection{Induction of EAE and visual acuity (VA) measurements in mice}

Before immunization, 6-week old C57BL/6 mice were housed under humidity and temperature-controlled conditions with 12-hour dark/ light cycle for two weeks. Mice then underwent baseline DBSI of optic nerves, followed acclimation in the facility for another 10-14 days before active immunization with myelin oligodendrocyte glycoprotein peptide $\left(\mathrm{MOG}_{35-55}\right)$. Immunization was performed over two sites on the flanks with the antigen emulsified in incomplete Freund's adjuvant (IFA), followed by intravenous injections of $200 \mathrm{ng}$ Pertussis toxin (List Laboratories, Campbell CA) on days 0 and 2. Sham-EAE was prepared on naïve mice with injection of IFA without antigen followed by $200 \mathrm{ng}$ of Pertussis toxin on days 0 and 2 (Fig. 1A).

The VA of mice was measured as previous described using the Virtual Optomotry System (Optomotry, Cerebral Mechanics, Inc.). Before immunization, normal VA was confirmed for each eye $(\mathrm{VA}=0.34 \pm 0.04 \mathrm{c} /$ $\mathrm{d}, \mathrm{n}=34$ eyes from 17 mice) at baseline. After immunization, blinded VA measurement was performed daily before the onset of ON (9-22 days post-immunization) which was defined as $\mathrm{VA} \leq 0.25 \mathrm{c} / \mathrm{d}$ in our previous study (Chiang et al., 2014; Lin et al., 2017, 2014, 2020), and then weekly till the end of the study (Fig. 1B).

\subsection{Fingolimod treatment}

Starting on the first day of onset of ON, mice were alternately assigned to either receive daily gavage of fingolimod $(n=9$ mice, $1 \mathrm{mg}$ /

A

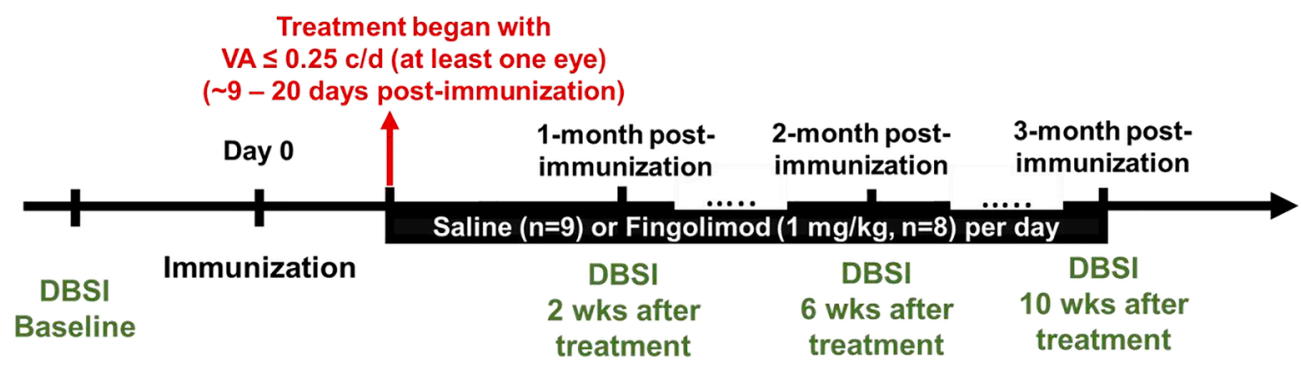

B

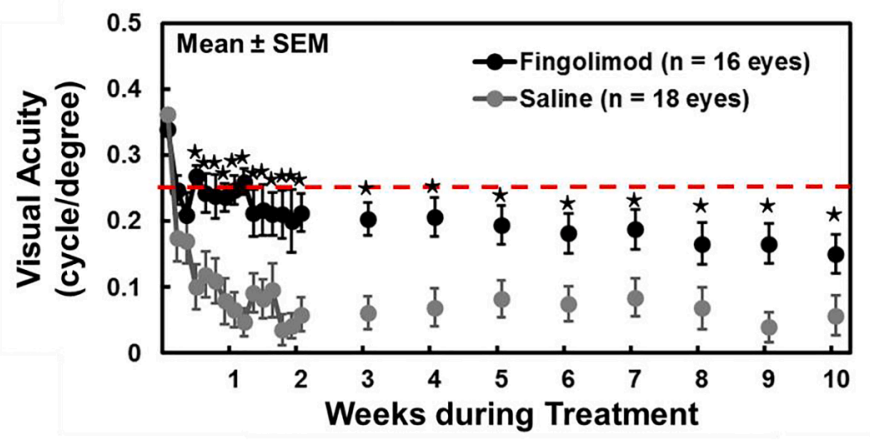

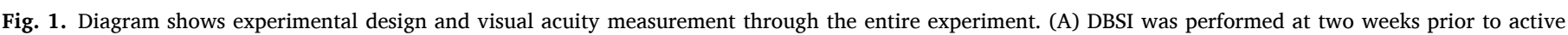

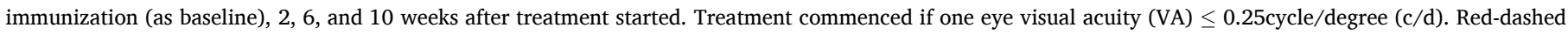

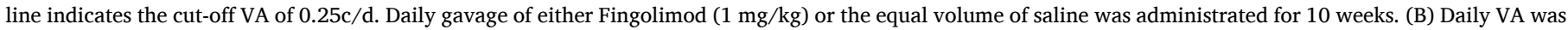

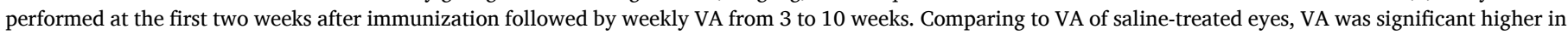

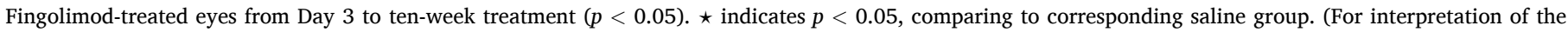
references to colour in this figure legend, the reader is referred to the web version of this article.) 
$\mathrm{kg}$, Cat No. S5002, Selleck Chemicals LLC) or the same volume of saline ( $\mathrm{n}=9$ mice) for 10 consecutive weeks. Mice were alternately assigned to receive fingolimod or saline until the 9 th pair. The final number in fingolimod- and saline-treated group was 8 and 9 EAE mice, respectively due to the lack of VA impairment or clinical signs during the course of assessment of the last EAE mouse assigned to fingolimod treatment.

\subsection{In vivo diffusion basis spectrum imaging (DBSI)}

In vivo DBSI was performed two weeks before immunization (baseline DBSI), and then at two, six and ten weeks after initiation of fingolimod treatment. DBSI were performed on a 4.7-T small-animal MRI scanner (Agilent/Varian) equipped with an Agilent/Magnex HD imaging gradient coil (Magnex/Agilent). Mice were anesthetized with isoflurane $/ \mathrm{O}_{2}[2 \%(\mathrm{vol} / \mathrm{vol})$ for induction followed by $0.7-0.8 \%$ for maintenance] throughout the imaging experiment. Body temperature was maintained at $37^{\circ} \mathrm{C}$ with a circulating warm water pad. A diffusion weighted mid-sagittal image was acquired to localize mouse optic nerves, followed by an oblique image plan perpendicular to the optic nerve to obtain the final image view using a multi-echo spin-echo diffusion-weighting sequence ( $\mathrm{Tu}$ et al., 2014). The final diffusionweighted MRI for DBSI analysis employed a 25-direction diffusionencoding scheme adding an extra $\mathrm{b}=0$ data as previously reported (Chiang et al., 2014) with the following acquisition parameters: TR = $1500 \mathrm{~ms}, \mathrm{TE}=35 \mathrm{~ms}, \mathrm{NE}=2$, inter-echo delay $=20.7 \mathrm{~ms}, \mathrm{FOV}=22.5 \times$ $22.5 \mathrm{~mm}^{2}$, matrix size $=192 \times 192$ (zero-filled to $384 \times 384$ ), slice thickness $=0.8 \mathrm{~mm}$, maximal b-value $=2,200 \mathrm{~s} / \mathrm{mm}^{2}$, total scan time $=$ $2 \mathrm{hr} .4 \mathrm{~min}$.

\subsection{Imaging analysis}

Data was processed with DBSI multi-tensor analysis packages developed in-house with Matlab. (Wang et al., 2015, 2011). The imaging data were modeled according to Equation 1:

$S_{k}=\sum_{i=1}^{N_{\text {Aniso }}} f_{i} e^{-\left|\vec{b}_{k}\right| \lambda_{\perp i}} e^{-\left|\vec{b}_{k}\right|\left(\lambda_{\| i}-\lambda_{\perp i}\right) \cos ^{2} \psi_{i k}}+\int_{a}^{b} f(D) e^{-\left|\vec{b}_{k}\right| D} d D(k=1,2,3, \cdots, 25)$

The quantities $S_{k}$ and $\left|\overrightarrow{b_{k}}\right|$ are the signal and $b$-value of the $k^{\text {th }}$ diffusion gradient, $N_{\text {Aniso }}$ is the number of anisotropic tensors (fiber tracts), $\Psi_{i k}$ is the angle between the $k^{\text {th }}$ diffusion gradient and the principal direction of the $i^{\text {th }}$ anisotropic tensor, $\lambda_{\| i}$ and $\lambda_{\perp i}$ are the axial and radial diffusivity of the $i^{\text {th }}$ anisotropic tensor, $f_{i}$ is the signal intensity fraction for the $i^{\text {th }}$ anisotropic tensor, and $a$ and $b$ are the low and high diffusivity limits for the isotropic diffusion spectrum (reflecting cellularity and edema) $f(D)$. For a coherent fiber bundle like the optic nerve, $N_{\text {Aniso }}=1$. DBSI derived $f_{i}$ represents the density of axons derived from retinal ganglion cells (fiber fraction) in the image voxel, taking into account of intra-voxel pathological and structural complications. Based on prior work, DBSI derived $\lambda_{\|}$and $\lambda_{\perp}$ reflect axon and myelin integrity respectively: $\downarrow \lambda_{\|}=$axonal injury and $\uparrow \lambda_{\perp}=$ demyelination. Our previous experimental findings suggest that the restricted isotropic diffusion fraction reflecting cellularity can be derived by the summation of $f(D)$ at $0 \leq \mathrm{ADC} \leq 0.6 \mu \mathrm{m}^{2} / \mathrm{ms}$. The summation of the remaining $f(D)$ at ADC $>0.6 \mu \mathrm{m}^{2} / \mathrm{ms}$ represents "non-restricted" isotropic diffusion, which denotes regions of tissue loss, increased inter-axonal space, vasogenic edema and CSF.

\subsection{Regions of interest (ROI)}

ROIs were manually drawn with ImageJ (https://imagej.nih.gov/ij/, $\mathrm{NIH}, \mathrm{US}$ ) at the center of each optic nerve on the diffusion-weighted image (the edge voxel of optic nerve were avoided), which corresponded to the diffusion gradient direction perpendicular to optic nerves, to minimize partial volume effects. ROIs were then transferred to the parametric maps to calculate the mean value for individual DBSI metrics. A separate ROI encompassing the whole optic nerve was drawn on cross-sectional images obtained with diffusion weighting gradient direction orthogonal to optic nerve (larger than the ROIs for other DBSI metrics). The ROI included the edge voxel of optic nerve. DBSI-derived fiber volume was calculated from the optic nerve volume (the entire ROI on DWI) multiplied by the corresponding DBSI fiber fraction. The dilution effect of inflammation and surrounding CSF is considered in the fiber volume computation in the manner (Lin et al., 2020).

\subsection{Histology analysis}

After the last DBSI scan, mice were killed and the optic nerves were collected and embedded in $2 \%$ agar gel (Blewitt et al., 1982), followed by paraffin embedding of the agar block. The paraffin blocks were cut in the axial plane at a thickness of $5-\mu \mathrm{m}$ for immunohistochemistry (IHC) staining, including SMI-31, SMI-312, MBP, and DAPI. The whole field of SMI-31, MBP, and DAPI stained images at $100 \times$ magnification with oil lens was captured with the same fluorescence light intensity and exposure time for each image. All captured images were converted to 8-bit gray scale and analyzed using threshold, edge enhancement, analyze particles and gray level watershed segmentation functions in ImageJ (https://imagej.nih.gov/ij/, NIH, US). Nucleus counts were performed by signal intensity threshold on DAPI staining (Lin et al., 2017, 2020).

\subsection{Statistical analysis}

Data were presented as mean \pm standard deviation. VA and MRI measurements were performed on each eye at baseline, end of two-, sixand ten-week treatment. Data were analyzed with a mixed random effects repeated measures model with side, time, treatment, and time by treatment interaction as fixed effects. Mouse within treatment was a random effect. Correlation of repeated-measures was estimated in a firstorder auto-regressive covariance structure. Variance components were estimated by group to account for differences in variance. Regression of MRI measurements on histology measures were performed with mixed random effect regression to account for two eyes measured for each mouse.

\section{Results}

\subsection{Visual acuity (VA) in fingolimod-treated mice was better than that in saline-treated mice}

There was no VA difference between fingolimod-treated $(0.34 \pm$ $0.03 \mathrm{c} / \mathrm{d}, \mathrm{n}=16$ eyes, from $8 \mathrm{EAE}$ mice $)$ and saline-treated groups $(0.36$ $\pm 0.04 \mathrm{c} / \mathrm{d}, \mathrm{n}=18$ eyes, from $9 \mathrm{EAE}$ mice) at baseline before treatment. Over the treatment period, the VA of fingolimod-treated mice was significantly better than saline-treated mice from 3 days to 10 weeks after treatment onset ( $p<0.05$, Fig. 1B).

\subsection{DBSI detected the reduced inflammation in fingolimod-treated optic} nerves

Representative DBSI non-restricted isotropic fraction (putatively reflecting vasogenic edema) and restricted isotropic fraction (putatively reflecting cellularity) maps of optic nerves were presented to demonstrate the evolution of these markers at baseline (before immunization), 2, 6, and 10 weeks after treatment (Fig. 2A).

In the group of saline-treated mice with $\mathrm{ON}$, non-restricted isotropic fraction of optic nerves significantly increased from baseline by $133 \%(p$ $=0.020), 198 \%(p=0.001)$, and $229 \%(p<0.001)$ at 2,6 , and 10 weeks after treatment, respectively (Fig. 2B). Restricted isotropic fraction of optic nerves significantly increased from the baseline by $201 \%(p<$ $0.001), 98 \%(p=0.070)$, and $164 \%(p=0.002)$ at 2,6 , and 10 weeks after placebo treatment, respectively (Fig. 2C). In the fingolimod 


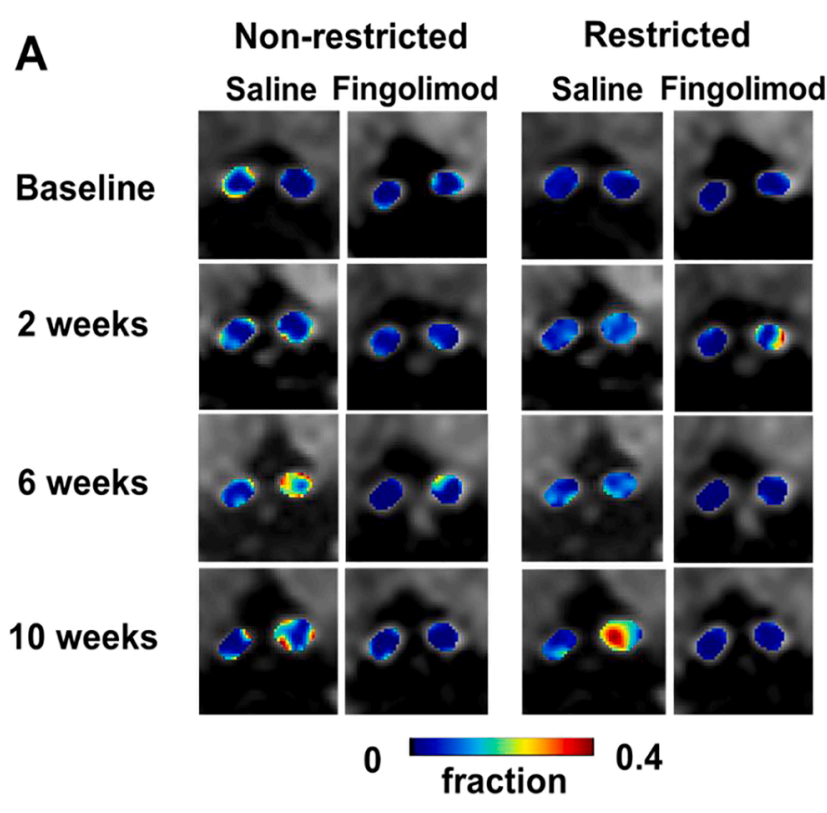

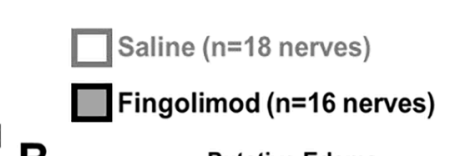

B

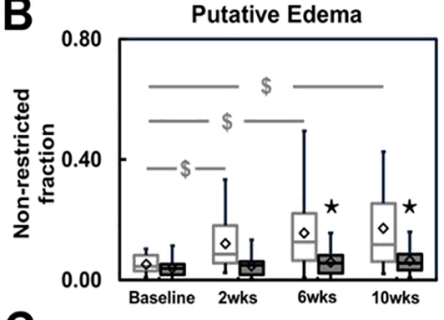

C

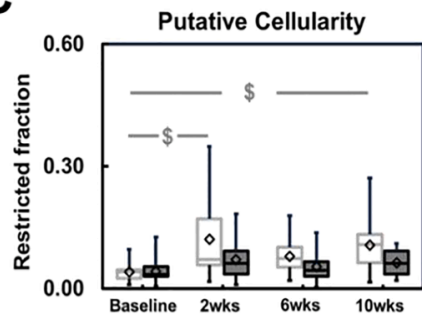

Fig. 2. DBSI-derived non-restricted fraction (putative edema) and restricted (putative cellularity) isotropic tensor fraction maps for representative saline- and fingolimod-treated optic nerves from baseline to 10 -week treatment (A). Comparing to baseline, fingolimod effectively suppressed putative inflammation markers, including non-restricted and restricted fractions, after treatment $(p<$ 0.05 , B and C). However, significant increase in saline-treated optic nerves from baseline was shown in non-restricted fraction at all time points $(p<0.05)$ and in restricted fraction at 2 and 10 (both $p<0.05$ ) but not at $6(p=0.06)$ weeks (B and C). Comparing to saline, significantly reduced non-restricted fraction at 6 and 10 weeks $(p<0.05)$ in fingolimod-treated optic nerves (B). Mild but not significant reduced restricted fraction was shown in fingolimod-treated optic nerve all the time (C). $\star$ indicates $p<0.05$, comparing between saline and fingolimod groups $\$$ indicates $p<0.05$, comparing to its baseline within group. treatment group, non-restricted isotropic fraction increased from baseline by $21 \%(p=0.740), 48 \%(p=0.440)$, and $63 \%(p=0.320)$ at 2,6 , and 10 weeks after treatment (Fig. 2B), while restricted isotropic fraction increased from baseline by $72 \%(p=0.180), 28 \%(p=0.590)$, and $51 \%(p=0.340)$ at 2,6 , and 10 weeks (Fig. 2 C).

In comparison to the saline-treated group, the fingolimod-treated mice exhibited significantly lower non-restricted isotropic fraction at 2 (60\% lower, $p=0.070$ ), 6 ( $62 \%$ lower, $p=0.020$ ) and 10 weeks $(63 \%$ lower, $p=0.010$ ) after treatment (Fig. 2B). Although the increase in restricted isotropic fraction was numerically greater for the placebo group, differences between the two treatment groups were not statistically significant.

\subsection{DBSI detected reduced axon loss in fingolimod-treated optic nerves}

Representative fiber fraction (the total diffusion signal from anisotropic axonal fiber bundles) maps of optic nerves at baseline, 2, 6, and 10 weeks after treatment were presented to demonstrate the evolution of fiber fraction (Fig. 3A).

In the saline-treated group, DBSI fiber fraction (putative marker of apparent axonal density) of the optic nerves decreased by $10 \%$ ( $p=$ $0.140), 24 \%(p=0.002)$ and $25 \%(p=0.003)$ at 2,6 , and 10 weeks, respectively, when compared to baseline before immunization (Fig. 3B). DWI-derived volumes of optic nerves decreased by $23 \%(p=0.004)$, $37 \%(p<0.001)$ and $25 \%(p=0.002)$ at 2,6 , and 10 weeks, respectively, from baseline before immunization (Fig. 3C). DBSI-derived axonal volume (putative marker of axonal content) decreased by $28 \%$

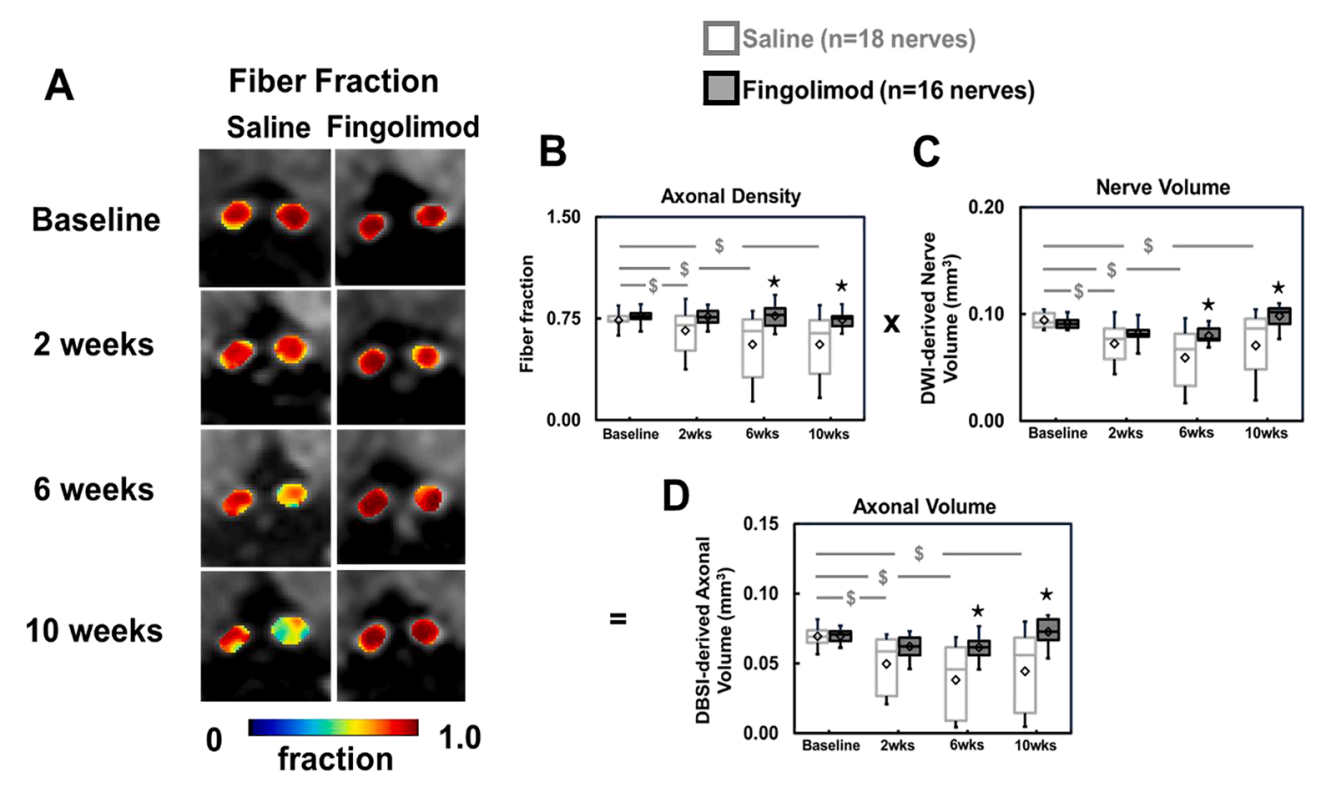

Fig. 3. DBSI-derived fiber fraction reflects total signal from anisotropic diffusion tensor components, reflecting axonal fiber density (A, B). Comparing to baseline, significantly decreased fiber fraction was detected at each time point in saline-treated group ( $p<0.05$ ) but not in fingolimod-treated optic nerves at all time points (B). Significantly reduced DWI-derived nerve volume was seen in saline-treated optic nerves $(p<0.05)$ but not in fingolimod-treated group from baseline to the rest of time points (C). To remove the confounding effects from inflammation, DBSI-derived axonal volume (D) was computed multiplying fiber fraction (B) by DWI-derived nerve volume (C) in each individual optic nerve. Comparing to baseline, significantly reduced DBSI-derived axonal volume was shown in saline-treated optic nerves $(p<0.05)$ but not in fingolimodtreated optic nerves at all time points (D). Comparing to saline group, fingolimodtreated optic nerves showed significantly higher axon volume at all time points (D), suggesting axonal prevention with fingolimod treatment. $\star$ indicates $\mathrm{p}<0.05$, comparing between saline and fingolimod 
$(p=0.006), 45 \%(\mathrm{p}<0.001)$, and $36 \%(p<0.001)$ at 2,6 , and 10 weeks, respectively, when compared to baseline before immunization (Fig. 3D). In the fingolimod-treated group, no significant difference from baseline was observed for DBSI fiber fraction, DWI-derived optic nerve volume, or DBSI axonal volume at any time point after treatment.

Compared to saline-treated mice, the fingolimod-treated group exhibited significantly higher DBSI fiber fraction at 6 weeks $(38 \%$ higher, $p=0.020$ ) and 10 weeks (Fig. 3B, 33\% higher, $p=0.030$ ), with significantly lower DWI-derived optic nerve volumes at 6 weeks (35\% higher, $p=0.02$ ) and at 10 weeks (39\% higher, $p=0.005$, Fig. $3 \mathrm{C}$ ). DBSI-derived axonal volumes in the fingolimod-treated optic nerves were higher than for saline-treated optic nerves at 2 weeks ( $25 \%$ higher, $p=0.400$ ), 6 weeks ( $62 \%$ higher, $p=0.020$ ) and 10 weeks ( $64 \%$ higher, $p=0.007$ ) after treatment commencement (Fig. 3D).

\subsection{DBSI detection of diminished injury to residual axons in fingolimod- treated optic nerves}

Fig. 4A shows representative DBSI axial diffusivity $\left(\lambda_{\|}\right)$and radial diffusivity $\left(\lambda_{\perp}\right)$ maps at baseline, 2, 6, and 10 weeks after treatment for each group. Compared to baseline, for saline-treated mice DBSI $\lambda_{\|}$ significantly decreased by $17 \%(p<0.001), 14 \%(p<0.001)$, and $12 \%$ $(p=0.001)$ at 2,6 , and 10 weeks (Fig. 4B), while DBSI $\lambda_{\perp}$ significantly increased by $45 \%(p=0.015), 62 \%(p=0.001)$ and $75 \%(p<0.001)$ at 2, 6, and 10 weeks (Fig. 4C), respectively. In the fingolimod treatment group, DBSI $\lambda_{\|}$decreased by $7 \%(p=0.040), 7 \%(p=0.033), 3 \%(p=$ 0.330 ) at $2,6,10$ weeks (Fig. 4B), while DBSI $\lambda_{\perp}$ increased from baseline by $14 \%(p=0.400), 15 \%(p=0.400)$ and $9 \%(p=0.600)$ at 2,6 and 10 weeks after treatment (Fig. 4C).

Compared the difference between groups (saline vs. fingolimod), fingolimod-treated group showed no significant differences on DBSI $\lambda_{\|}$ for all the time points while DBSI $\lambda_{\perp}$ decreased by $38 \%$ at 10 weeks ( $\mathrm{p}=$ 0.010, Fig. 4C).

\subsection{Immunohistochemistry (IHC) staining of optic nerves}

Mice were euthanized at the end of the treatment period for histologic assessment of the optic nerves. IHC staining of total neurofilaments (SMI-312, stains both injured and intact axons), phosphorylated neurofilaments (SMI-31, stains intact axons), and myelin basic protein (MBP, stains myelin) revealed that the saline-treated group exhibited less staining for SMI-312 (Fig. 5A), MBP (Fig. 5D), and SMI-31 (Fig. 5G), when compared to fingolimod-treated group (Fig. 5B, E, H). The apparent optic nerve sizes of saline-treated group were smaller than those of fingolimod-treated group (Fig. 5A-I). However, compared to one representative wildtype (WT) optic nerves (10-week-old C57BL/6 female mouse optic nerve), fingolimod-treated group showed enlarged staining spots (Fig. 5b, e, h; blue arrows), suggesting swollen axons or myelin debris aggregation. Moreover, increased positive DAPI staining areas (cell nuclei) were also seen in both saline and fingolimod treatment groups compared to WT optic nerve, suggesting increased cellularity (Fig. 5J and $\mathrm{K}$ ).

DBSI $\lambda_{\|}$, DBSI $\lambda_{\perp}$, DBSI-derived axon volume, and DBSI restricted/ non-restricted fractions correlated well with SMI-31 area fraction (Fig. 6A), MBP area fraction (Fig. 6B), SMI-312 area fraction (Fig. 6C) and DAPI density (Fig. 6D/E), respectively in the ON-affected optic nerves.

\section{Discussion}

Understanding of the evolution of chronic human CNS diseases over time and response to therapies has been hindered by inability to access CNS specimens to examine longitudinally. In this study, we hypothesized that DBSI is capable to assess the efficacy of fingolimod treatment in an animal model of ON. The optic nerve was chosen for examination because it is a frequent site of inflammatory demyelination in MS and related diseases. Moreover, the volume of the optic nerve can be measured, allowing precise measurement of axonal content, even in the presence of inflammation and edema. Previously, DTI has played a significant role to noninvasively identify axonal injury and demyelinaiton in MS subjects (Kuchling et al., 2017; Naismith et al., 2009, 2012b; Smith et al., 2011) or its animal models (Sun et al., 2007). However, single-tensor DTI-determined axonal injury or demyelination may be confounded by coexisting inflammation limiting its ability to accurately assess the severity of axonal loss, a hallmark pathology of disease progression. DBSI is a data-driven approach modeling diffusionweighted MRI signals as a linear combination of multiple anisotropic and isotropic tensors to resolve the underlying structural and pathological complexity. Thus, DBSI noninvasively quantifies coexisting pathologies over time and quantitatively assesses axonal loss in fingolimod (an FDA-approved oral medication for MS) or saline-treated optic nerves.

Optic neuritis, a common occurrence in people with MS, can lead to irreversible visual impairment (Galetta et al., 2015). Fingolimod is approved for patients with RRMS. However, its neuroprotective effects remain uncertain (Ambrosius et al., 2017). Rau et al. reported apoptotic

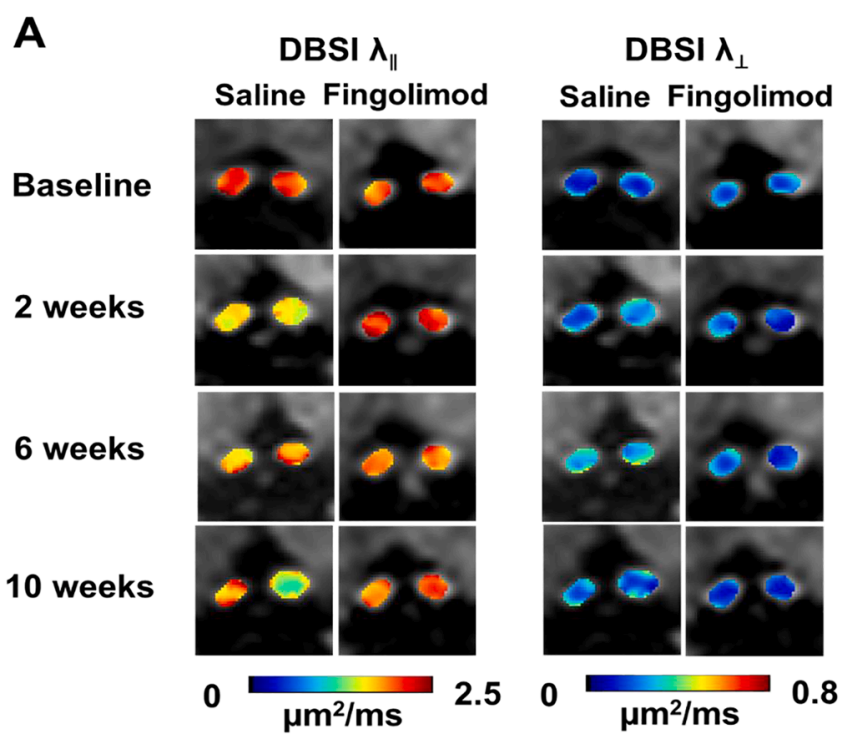

Saline ( $n=18$ nerves)

$\square$ Fingolimod ( $n=16$ nerves)
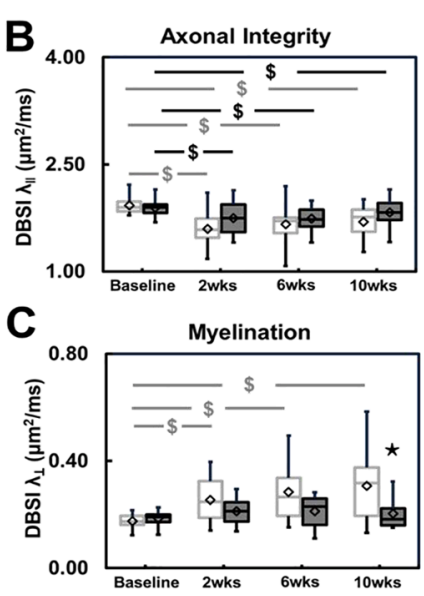

Fig. 4. DBSI-derived axial $\left(\lambda_{\|}\right)$and radial $\left(\lambda_{\perp}\right)$ diffusivity maps of representative salineand fingolimod-treated optic nerves from baseline to 10-week treatment (A) were derived from anisotropic diffusion tensor components. Comparing to baseline, significantly decreased DBSI- $\lambda_{\|}$was detected at all time points in saline-treated optic nerves (B, $\mathrm{p}<0.05)$. In contrast, decreased DBSI- $\lambda_{\|}$ was only seen at 2 and 6 weeks in fingolimod-treated optic nerves $(B, p<$ $0.05)$. Significantly increased DBSI- $\lambda_{\perp}$ was detected in saline-treated $(\mathrm{p}<0.05$ ) but not in fingolimod-treated optic nerves at all time points (C). The results showed intermittent axonal injury during fingolimod treatment. After 10-week fingolimod treatment, DBSI- $\lambda_{\|}$ and DBSI- $\lambda_{\perp}$ were not different from their baseline values (B, C). $\star$ indicates $\mathrm{p}<0.05$, comparing between saline and fingolimod groups $\$$ indicates $\mathrm{p}<0.05$, comparing to its baseline within group. 


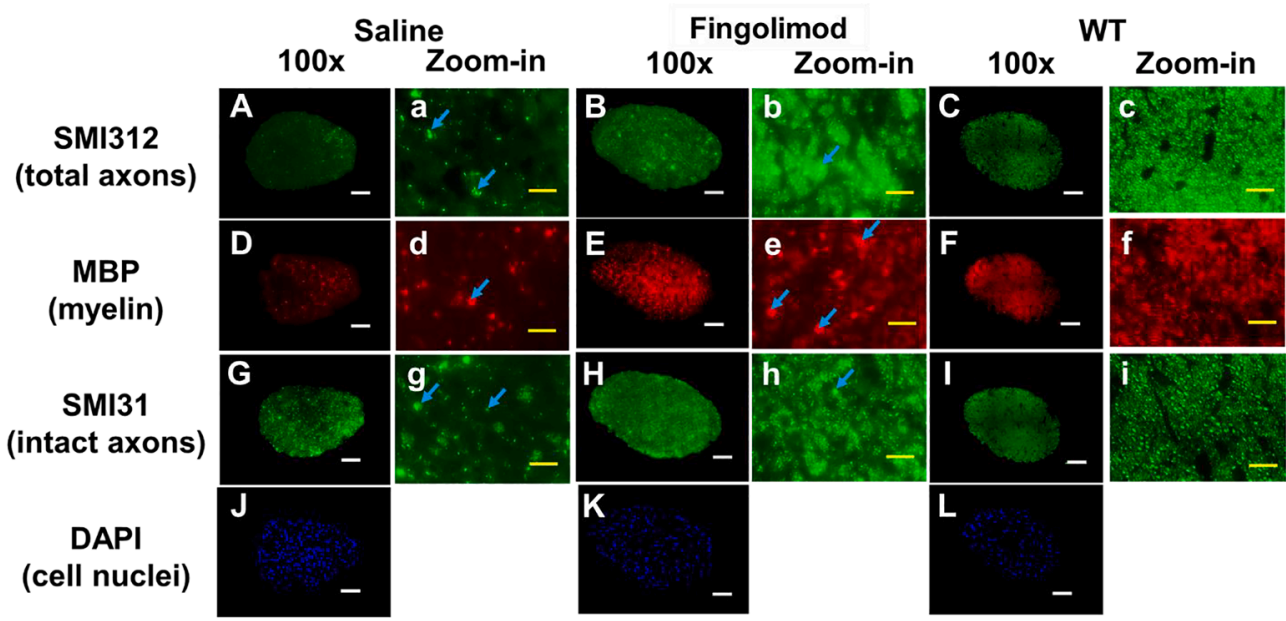
fingolimod-treated (K) and wild-type (L) optic nerves.White scale bar: $50 \mu \mathrm{m}$ Yellow scale bar: $10 \mu \mathrm{m}$. (For interpretation of the references to colour in this figure legend, the reader is referred to the web version of this article.) chemical staining images of total neurofilaintact axons, A-C), myelin basic protein (J) suggested highest cellularity than
Fig. 5. Representative $100 \times$ immunohistoment (SMI-312, staining both injured and (MBP, assessing myelin, D-E), phosphorylated neurofilament (SMI-31, reflecting intact axons, G-I), and 4', 6-dianidino-2-phenylindole (DAPI, detecting nuclei, $\mathrm{J}-\mathrm{L}$ ) from saline-, and fingolimod-treated optic nerves at 10 weeks after treatment. Comparing to wild-type (WT) optic nerves, saline-treated optic nerves showed lowest staining intensity in SMI-312 (A), MBP (D), and SMI-31 (G), suggesting axonal loss, demyelination, and axonal injury, respectively. In contrst, moderate lower staining intensity in SMI-312 (B), MBP (E), and SMI-31(H), suggesting mild axon and myelin injury were assessed in fingolimod-treated optic nerves. Blue arrows indicated swelling axon and myelin. Strongest DAPI stains in saline-treated optic nerve Fig. 6. Mixed random-effect regression analysis for the correlation between DBSI parameters and IHC biomarkers. SMI-31 counts and MBP area fraction (the ratio of positive staining counts and total tissue area), SMI-312 area (absolute value of positive staining counts), and DAPI counts were statistically significant associated with DBSI$\lambda_{\|}$(A), DBSI- $\lambda_{\perp}$ (B), DBSI-derived axonal volume (C), DBSI restricted isotropic fraction (D), and DBSI non-restricted isotropic fraction (E), suggesting that in vivo DBSI quantitatively reflected the complicated pathologies including axonal injury, demyelination, axonal loss, and cell infiltration.

\section{E}

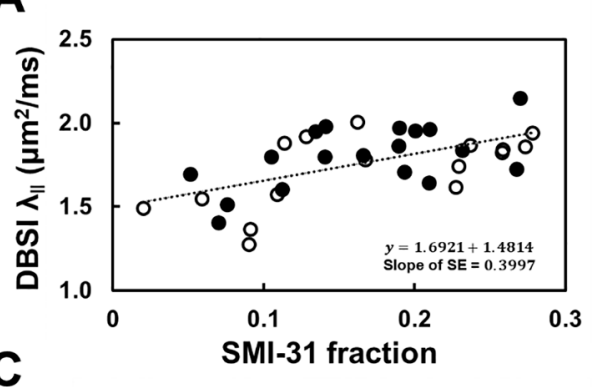

D
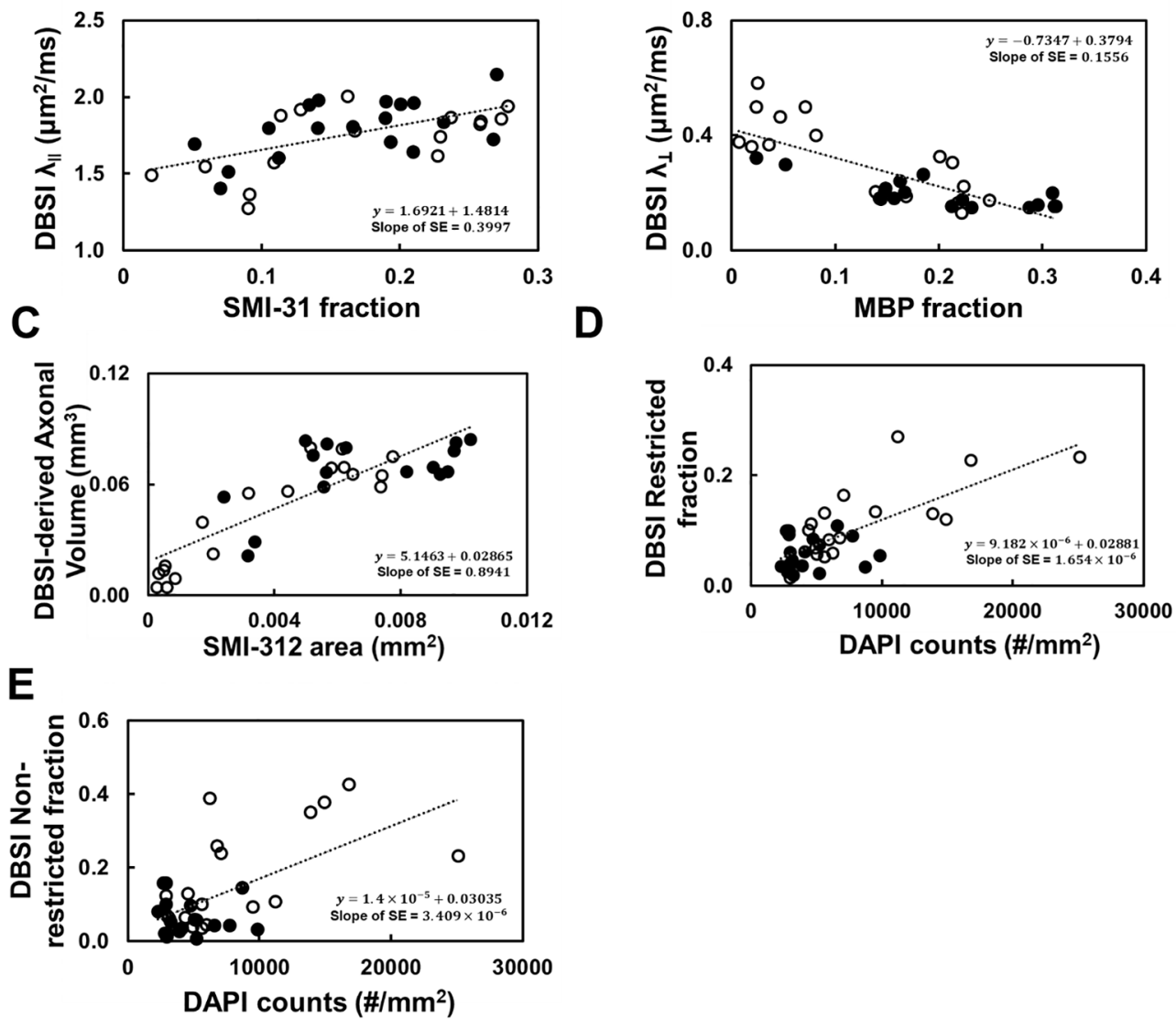

cell death of retinal ganglion cells in rats with EAE that were treated with fingolimod, although axonal preservation in optic nerves was seen by histological data (Rau et al., 2011). An et al. reported axonal preservation in optic nerves by histological validation and moderate visual dysfunction (better than non-treated EAE mice but worse than naïve control mice) by optometry (An et al., 2013). However, both studies could only rely upon end-point histological results at the conclusion of study to evaluate whether fingolimod is axonal protective. In the current study, DBSI detected improved transient inflammation (via the putative inflammatory markers: restricted fraction $\rightarrow$ inflammatory cellularity 
and non-restricted fraction $\rightarrow$ vasogenic edema) and axonal pathologies (via putative DBSI axonal injury and loss markers) in fingolimod-treated optic nerves. Results suggest axonal protection of fingolimod could be secondary to reduced inflammation.

We observed that daily oral fingolimod markedly reduced inflammation, evidenced by the decrease in DBSI restricted isotropic fraction (reflecting cellular inflammation) and non-restricted isotropic fraction (reflecting vasogenic edema), in accordance with the action of fingolimod to sequester lymphocytes in secondary lymphoid tissue and thus inhibit lymphocytes from entering the CNS (Zhang et al., 2015b). Additionally, we also observed that both decreased $\lambda_{\|}$(putative axonal injury, Fig. 4B) and increased $\lambda_{\perp}$ (putative demyelination, Fig. 4C) were significantly improved in mice treated with fingolimod versus those treated with saline. Mice were euthanized and optic nerves were examined histologically at the conclusion of 10-week treatment. As previously reported (Lin et al., 2017, 2020) DBSI longitudinally monitored disease progression. After 10 weeks of fingolimod therapy, reduced inflammation, less demyelination, and diminished axonal injury of optic nerves was confirmed by IHC, suggesting DBSI is capable of detecting long-term anti-inflammatory and neuroprotective effects with fingolimod treatment. The histological findings were consistent with DBSI-derived putative imaging biomarkers of optic nerve pathologies. Following increased $\lambda_{\perp}$ (demyelination) in optic nerves of EAE mice with ON, we noted the subsequent reduction of DBSI $\lambda_{\perp}$ after 10 weeks of fingolimod but not placebo treatment, suggesting remyelination could have occurred in those mice treated with fingolimod. There is no direct histological data to validate in this study, but the profile of DBSI-derived $\lambda_{\perp}$ was consistent with other reports (Yazdi et al., 2020; Zhang et al., 2015a).

Limitations of the current study include that we performed histological validation at the last time point, 10 weeks after treatment commenced. Definitive demonstration of the evolution of the underlying optic nerve pathologies may require further cross-sectional histological validation studies. Second, this proof-of-concept study may be limited due to the small sample size (17 mice divided between fingolimod and saline treatment groups). Although each mouse was imaged 4 times, generating 68 imaging datasets thus increasing the power of the study, the limitation of small number of animals studied needs to be kept in mind when drawing a conclusion. Third, visual function is not specific to optic nerve pathologies since EAE (and MS) can affect other parts of the visual system, such as retinal ganglion cells (Shindler et al., 2006). Damage in regions other than the optic nerves was not taken into consideration in the present study. Finally, not all DBSI pathologic metrics have corresponding IHC markers. For example, non-restricted fraction is a putative marker of vasogenic edema in vivo, associated with inflammation in EAE. However, postmortem histology is not suitable for validating this metric due to fixation and paraffin embedding for IHC analysis although indirect evidence does support that non-restricted fraction is associated with inflammation (Fig. 6E, correlation between non-restricted fraction and DAPI).

In conclusion, our preclinical study demonstrates that DBSI-based biomarkers reflect underlying pathologic changes and, importantly, therapeutic efficacy of an established treatment. Moreover, the results strongly suggest that DBSI can detect and quantitate remyelination following demyelination. These data support that DBSI, a noninvasive contrast agent-free MR technique, offers new possibilities for precise clinical diagnosis as well as assessing disease evolution and treatment responses in CNS inflammatory demyelinating diseases, such as MS.

\section{Declaration of Competing Interest}

The authors declare that they have no known competing financial interests or personal relationships that could have appeared to influence the work reported in this paper.

\section{Acknowledgement}

The authors thank Mr. Bob Mikesell for excellent technical assistance. This study was supported in part by the grants from National Institute of Health R01-NS047592 (SKS), P01-NS059560 (AHC), U01EY025500 (SKS), National Multiple Sclerosis Society (NMSS) RG 4549A4/1 (SKS), RG1701-26617 (SKS), FG-1507-05315 (THL), Department of Defense Idea Award W81XWH-12-1-0457 (SKS), the National Natural Science Foundation of China 81971574 (RY), the Guangzhou Science and Technology Project, P.R. China 202002030268 (RY) and Natural Science Foundation of Guangdong Province in China 2018A030313282 (RY). the Natural Science Foundation of Jiangxi, P. R. China 20202BABL216038 (JZ), the Science and Technology Project of Jiangxi Health Committee 202110018 (JZ), and the Science and Technology Research Project of Jiangxi Provincial Department of Education GJJ180133 (JZ). AHC was supported in part by the Manny and Rosalyn Rosenthal-Dr. John L. Trotter MS Center Chair in Neuroimmunology of Barnes-Jewish Hospital Foundation.

\section{References}

Ambrosius, B., Pitarokoili, K., Schrewe, L., Pedreiturria, X., Motte, J., Gold, R., 2017. Fingolimod attenuates experimental autoimmune neuritis and contributes to Schwann cell-mediated axonal protection. J. Neuroinflamm. 14, 92.

An, X.M., Kezuka, T., Usui, Y., Matsunaga, Y., Matsuda, R., Yamakawa, N., Goto, H., 2013. Suppression of experimental autoimmune optic neuritis by the novel agent fingolimod. J. Neuroophthalmol. 33, 143-148.

Barkhof, F., 2002. The clinico-radiological paradox in multiple sclerosis revisited. Curr, Opin. Neurol. 15 (3), 239-245.

Blewitt, E.S., Pogmore, T., Talbot, I.C., 1982. Double Embedding in Agar Paraffin Wax as an Aid to Orientation of Mucosal Biopsies. Journal of Clinical Pathology 35, 365365.

Chiang, C.-W., Wang, Y., Sun, P., Lin, T.-H., Trinkaus, K., Cross, A.H., Song, S.-K., 2014. Quantifying white matter tract diffusion parameters in the presence of increased extra-fiber cellularity and vasogenic edema. Neuroimage 101, 310-319.

Costello, F., Coupland, S., Hodge, W., Lorello, G.R., Koroluk, J., Pan, Y.I., Freedman, M. S., Zackon, D.H., Kardon, R.H., 2006. Quantifying axonal loss after optic neuritis with optical coherence tomography. Ann. Neurol. 59 (6), 963-969.

Davis, F.A., 2014. The clinico-radiological paradox in multiple sclerosis: novel implications of lesion size. Mult Scler 20 (4), 515-516.

Gajamange, S., Raffelt, D., Dhollander, T., Lui, E., van der Walt, A., Kilpatrick, T., Fielding, J., Connelly, A., Kolbe, S., 2018. Fibre-specific white matter changes in multiple sclerosis patients with optic neuritis. Neuroimage Clin. 17, 60-68.

Galetta, S.L., Villoslada, P., Levin, N., Shindler, K., Ishikawa, H., Parr, E., Cadavid, D., Balcer, L.J., 2015. Acute optic neuritis: unmet clinical needs and model for new therapies. Neurol. Neuroimmunol. Neuroinflamm. 2 (4), e135. https://doi.org/ 10.1212/NXI.0000000000000135.

Hickman, S.J., Toosy, A.T., Miszkiel, K.A., Jones, S.J., Altmann, D.R., MacManus, D.G., Plant, G.T., Thompson, A.J., Miller, D.H., 2004. Visual recovery following acute optic neuritis-a clinical, electrophysiological and magnetic resonance imaging study. J. Neurol. 251, 996-1005.

Hickman, S.J., Wheeler-Kingshott, C.A., Jones, S.J., Miszkiel, K.A., Barker, G.J., Plant, G. T., Miller, D.H., 2005. Optic nerve diffusion measurement from diffusion-weighted imaging in optic neuritis. AJNR Am. J. Neuroradiol. 26, 951-956.

Jackson, A., Sheppard, S., Laitt, R.D., Kassner, A., Moriarty, D., 1998. Optic neuritis: MR imaging with combined fat- and water-suppression techniques. Radiology 206 (1), 57-63.

Kavaliunas, A., Manouchehrinia, A., Stawiarz, L., Ramanujam, R., Agholme, J., Hedström, A.K., Beiki, O., Glaser, A., Hillert, J., 2017. Importance of early treatment initiation in the clinical course of multiple sclerosis. Mult. Scler. 23 (9), 1233-1240.

Kuchling, J., Brandt, A.U., Paul, F., Scheel, M., 2017. Diffusion tensor imaging for multilevel assessment of the visual pathway: possibilities for personalized outcome prediction in autoimmune disorders of the central nervous system. EPMA J. 8 (3), 279-294.

Leray, E., Yaouanq, J., Le Page, E., Coustans, M., Laplaud, D., Oger, J., Edan, G., 2010. Evidence for a two-stage disability progression in multiple sclerosis. Brain 133 (7), 1900-1913.

Lin, T.H., Chiang, C.W., Perez-Torres, C.J., Sun, P., Wallendorf, M., Schmidt, R.E., Cross, A.H., Song, S.K., 2017. Diffusion MRI quantifies early axonal loss in the presence of nerve swelling. J. Neuroinflamm. 14, 78.

Lin, T.-H., Kim, J.H., Perez-Torres, C., Chiang, C.-W., Trinkaus, K., Cross, A.H., Song, S.K., 2014. Axonal transport rate decreased at the onset of optic neuritis in EAE mice. Neuroimage 100, 244-253.

Lin, T.H., Zhan, J., Song, C., Wallendorf, M., Sun, P., Niu, X., Yang, R., Cross, A.H., Song, S.K., 2020. Diffusion basis spectrum imaging detects axonal loss after transient dexamethasone treatment in optic neuritis mice. Front. Neurosci. 14, 592063.

Montalban, X., Gold, R., Thompson, A.J., Otero-Romero, S., Amato, M.P., Chandraratna, D., Clanet, M., Comi, G., Derfuss, T., Fazekas, F., Hartung, H.P., Havrdova, E., Hemmer, B., Kappos, L., Liblau, R., Lubetzki, C., Marcus, E., Miller, D. H., Olsson, T., Pilling, S., Selmaj, K., Siva, A., Sorensen, P.S., Sormani, M.P., 
Thalheim, C., Wiendl, H., Zipp, F., 2018. ECTRIMS/EAN guideline on the pharmacological treatment of people with multiple sclerosis. Mult. Scler. 24 (2), 96-120.

Naismith, R.T., Xu, J., Tutlam, N.T., Lancia, S., Trinkaus, K., Song, S.K., Cross, A.H., 2012a. Diffusion tensor imaging in acute optic neuropathies: predictor of clinical outcomes. Arch. Neurol. 69, 65-71.

Naismith, R.T., Xu, J., Tutlam, N.T., Snyder, A., Benzinger, T., Shimony, J., Shepherd, J. Trinkaus, K., Cross, A.H., Song, S.-K., 2009. Disability in optic neuritis correlates with diffusion tensor-derived directional diffusivities. Neurology 72 (7), 589-594.

Naismith, R.T., Xu, J.Q., Tutlam, N.T., Lancia, S., Trinkaus, K., Song, S.K., Cross, A.H., 2012b. Diffusion tensor imaging in acute optic neuropathies predictor of clinical outcomes. Arch. Neurol. 69, 65-71.

Rahim, T., Becquart, P., Baeva, M.E., Quandt, J., 2018. Expression of the neuroprotective protein aryl hydrocarbon receptor nuclear translocator 2 correlates with neuronal stress and disability in models of multiple sclerosis. J. Neuroinflamm. 15, 270.

Rau, C.R., Hein, K., Sättler, M.B., Kretzschmar, B., Hillgruber, C., McRae, B.L., Diem, R., Bähr, M., 2011. Anti-inflammatory effects of FTY720 do not prevent neuronal cell loss in a rat model of optic neuritis. Am. J. Pathol. 178 (4), 1770-1781.

Rocca, M.A., Filippi, M., 2007. Functional MRI in multiple sclerosis. J. Neuroimaging 17 (Suppl 1), 36S-41S.

Scalfari, A., Neuhaus, A., Degenhardt, A., Rice, G.P., Muraro, P.A., Daumer, M., Ebers, G. C., 2010. The natural history of multiple sclerosis: a geographically based study 10 : relapses and long-term disability. Brain 133, 1914-1929.

Shindler, K.S., Guan, Y., Ventura, E., Bennett, J., Rostami, A., 2006. Retinal ganglion cell loss induced by acute optic neuritis in a relapsing model of multiple sclerosis. Mult. Scler. 12 (5), 526-532.

Shirani, A., Sun, P., Trinkaus, K., Perantie, D.C., George, A., Naismith, R.T., Schmidt, R. E., Song, S.-K., Cross, A.H., 2019. Diffusion basis spectrum imaging for identifying pathologies in MS subtypes. Ann. Clin. Transl. Neurol. 6 (11), 2323-2327.

Smith, S.A., Williams, Z.R., Ratchford, J.N., Newsome, S.D., Farrell, S.K., Farrell, J.A.D., Gifford, A., Miller, N.R., van Zijl, P.C.M., Calabresi, P.A., Reich, D.S., 2011. Diffusion tensor imaging of the optic nerve in multiple sclerosis: association with retinal damage and visual disability. AJNR Am. J. Neuroradiol. 32 (9), 1662-1668.
Sun, P., George, A., Perantie, D.C., Trinkaus, K., Ye, Z., Naismith, R.T., Song, S.K., Cross, A.H., 2020. Diffusion basis spectrum imaging provides insights into MS pathology. Neurol Neuroimmunol Neuroinflamm 7.

Sun, S.-W., Liang, H.-F., Schmidt, R.E., Cross, A.H., Song, S.-K., 2007. Selective vulnerability of cerebral white matter in a murine model of multiple sclerosis detected using diffusion tensor imaging. Neurobiol. Dis. 28 (1), 30-38.

Torkildsen, Ø., Myhr, K.-M., Bø, L., 2016. Disease-modifying treatments for multiple sclerosis - a review of approved medications. Eur. J. Neurol. 23 (S1), 18-27.

Tu, T.-W., Budde, M.D., Xie, M., Chen, Y.-J., Wang, Q., Quirk, J.D., Song, S.-K., 2014 Phase-aligned multiple spin-echo averaging: a simple way to improve signal-to-noise ratio of in vivo mouse spinal cord diffusion tensor image. Magn. Reson. Imaging 32 (10), 1335-1343.

Wang, X., Cusick, M.F., Wang, Y., Sun, P., Libbey, J.E., Trinkaus, K., Fujinami, R.S., Song, S.-K., 2014. Diffusion basis spectrum imaging detects and distinguishes coexisting subclinical inflammation, demyelination and axonal injury in experimental autoimmune encephalomyelitis mice. NMR Biomed. 27 (7), 843-852.

Wang, Y., Sun, P., Wang, Q., Trinkaus, K., Schmidt, R.E., Naismith, R.T., Cross, A.H., Song, S.-K., 2015. Differentiation and quantification of inflammation, demyelination and axon injury or loss in multiple sclerosis. Brain 138 (5), 1223-1238.

Wang, Y., Wang, Q., Haldar, J.P., Yeh, F.C., Xie, M., Sun, P., Tu, T.W., Trinkaus, K., Klein, R.S., Cross, A.H., Song, S.K., 2011. Quantification of increased cellularity during inflammatory demyelination. Brain 134, 3590-3601.

Yazdi, A., Ghasemi-Kasman, M., Javan, M., 2020. Possible regenerative effects of fingolimod (FTY720) in multiple sclerosis disease: An overview on remyelination process. J. Neurosci. Res. 98 (3), 524-536.

Ye, Z., George, A., Wu, A.T., Niu, X., Lin, J., Adusumilli, G., Naismith, R.T., Cross, A.H., Sun, P., Song, S.-K., 2020. Deep learning with diffusion basis spectrum imaging for classification of multiple sclerosis lesions. Ann. Clin. Transl. Neurol. 7 (5), 695-706.

Zhang, J., Zhang, Z.G., Li, Y.i., Ding, X., Shang, X., Lu, M., Elias, S.B., Chopp, M., 2015. Fingolimod treatment promotes proliferation and differentiation of oligodendrocyte progenitor cells in mice with experimental autoimmune encephalomyelitis. Neurobiol. Dis. 76, 57-66. 